\title{
Patología dual: integrando la salud mental
}

\author{
MD. Néstor Szerman \\ Hospital Universitario Gregorio Marañón, Madrid, España \\ Presidente de la Sección de Patología Dual de la Asociación Mundial de Psiquiatría (WPA) \\ Secretario de la Asociación Mundial de Patología Dual (WADD)
}

El término "patología dual" se emplea, en el campo de la salud mental, para identificar a las personas que sufren de un trastorno adictivo y otro mental. Estos problemas pueden presentarse de forma simultánea o secuencial a lo largo del ciclo vital (Szerman \& Martinez-Raga, 2015).

En la década de los ochenta del siglo pasado, los trastornos mentales fueron reconocidos como enfermedades y su atención fue integrada a los sistemas de salud. Al mismo tiempo, los trastornos adictivos fueron también identificados como enfermedades mentales y se incluyeron en diversas clasificaciones internacionales, como el Manual Diagnóstico y Estadístico de los Trastornos Mentales (DSM), de la Asociación Americana de Psiquiatría.

Sin embargo, las personas que padecían algún trastorno adictivo quedaron excluidas de la atención regularizada, y su tratamiento se relegó y desplazó a redes diferenciadas, con prácticas en ocasiones marginadas y no siempre validadas por la comunidad científica. Esta separación conceptual y asistencial ha propiciado que muchos pacientes sean tratados de manera inadecuada $y$, en consecuencia, que se presente el efecto conocido como "síndrome de la puerta equivocada", que tiene lugar cuando el paciente no encuentra la "puerta" idónea que le brinde la atención y el tratamiento adecuados para sus padecimientos, con el consecuente aumento de la morbilidad y la mortalidad.

Parte de la consideración sobre la relevancia de la patología dual deriva de la investigación epidemiológica, que en últimas décadas ha dejado claro que las adicciones frecuentemente cursan con otros trastornos mentales (Lev-Ran, Imtiaz, Rehm, \& Le Foll, 2013), entre los que se pueden incluir rasgos de personalidad anómalos, estados de enfermedad mental y alteraciones cognitivas. Sin embargo, los estudios epidemiológicos sobre las adicciones, por lo regular transversales, infra- diagnostican estos trastornos durante el ciclo vital. Estudios que tampoco consideran las adicciones comportamentales o sin sustancia -como el trastorno por juego u otros-, reconocidas ahora como equivalentes, por sus semejanzas clínicas y neurobiológicas con los trastornos adictivos causados por drogas. Hay que recordar que también estas adicciones se ponen en marcha sólo en personas vulnerables y con otros trastornos mentales (Chamberlain, Stochl, Redden, Odlaug, \& Grant, 2017).

Nos encontramos así, ante el hecho de que la separación entre la "salud mental" y las "adicciones", en algunos casos, niega la condición de enfermedad mental a los trastornos por uso de sustancias, lo que obstaculiza su reconocimiento. En los últimos años, gracias a la consideración de la adicción como un trastorno mental/cerebral, se han desarrollado, y en palabras de la doctora Nora Volkow, directora del National Institute on Drug Abuse (NIDA), "medidas preventivas, abordajes terapéuticos y políticas de salud pública eficaces para su abordaje; aunque esta consideración continúa siendo cuestionada por corrientes anticientíficas".

El reconocimiento de la adicción y otros trastornos psiquiátricos como trastornos mentales se debe, entre otros motivos, al progresivo conocimiento de los mecanismos neurobiológicos que subyacen a estas conductas, los mismos que la investigación en neurociencias ha comenzado a aclarar. "El concepto de adicción como un trastorno de la mente/cerebro desafía valores profundamente arraigados en la opinión pública, sobre autodeterminación y responsabilidad personal que señalan el uso de drogas por estas personas adictas como un acto voluntario y hedonista" (Volkow, Koob, \& McLellan, 2016).

La opinión de muchos expertos no distingue el consumo de sustancias, de la conducta adictiva; los factores sociales ponen en contacto a la población con las drogas o sustancias, pero son los factores individuales,

\section{Autor de correspondencia:}

MD. Néstor Szerman.

Correo electrónico: contacto@nestorszerman.com

DOI: 10.28931/riiad.2017.2.01 
los determinantes de la vulnerabilidad al uso compulsivo y la adicción (NIDA, 2007). Ésta no se produce sólo por los efectos reforzantes de las sustancias sobre el Sistema de Recompensa Cerebral, sino por otros mecanismos de vulnerabilidad -aun no suficientemente dilucidados- que separan de forma clara al uso recreativo del uso compulsivo. Por tanto, sólo una minoría de los consumidores de sustancias o personas que "juegan" tiene una adicción y quienes la padece, no la eligen (Belin, Belin-Rauscent, Everitt, \& Dalley, 2016).

Además, es conocido, pero con frecuencia ignorado, que el efecto de las sustancias con capacidad adictiva es diferente -y en ocasiones antagónico- en distintos grupos de personas, lo que incorpora a la patología dual, el concepto "Medicina de precisión" para tratar a los pacientes (Perna \& Nemeroff, 2017). Por ejemplo, un estimulante como la cafeína permite mantener despiertos a algunos individuos y ayuda a dormir mejor a otros. La cocaína, por otro lado, mejora la impulsividad en personas con rasgos impulsivos y la empeora en otros; esto ha dado lugar al comienzo del estudio de los rasgos de personalidad como "endofenotipos" que podrían preceder el inicio de una adicción.

La patología dual abarca estas y otras consideraciones, mientras las evidencias científicas actuales respaldan la postura de que no se trata de dos trastornos diferentes, sino de distintas manifestaciones clínicas, originadas en bases neurobiológicas comunes, que interactúan y se presentan según circunstancias individuales y medioambientales, de forma conjunta o secuencial, indistintamente de cual se manifieste primero (Szerman et al., 2013).

Así, el enfoque de la patología dual permite un tratamiento bio-psico-social personalizado que orienta el tratamiento hacia las personas y no hacia las sustancias.

Los pacientes con trastornos mentales, incluidas las adicciones, deben tener acceso a un único modelo asistencial multidisciplinar, que integre y coordine tanto la red de salud mental como la red de atención de las adicciones. Todo paciente tiene derecho a ser evaluado por profesionales expertos y a recibir una asistencia integral en patología dual, basada en las evidencias científicas (Sociedad Española de Patología Dual, 2017). La existencia de dos redes asistenciales no es eficaz ni eficiente y deja a muchos pacientes sin diagnóstico de patología dual y, por tanto, sin acceso a un tratamiento integral, como puso de manifiesto el estudio epidemiológico Madrid (Arias et al., 2013).

El concepto de patología dual, basado en la neurociencia-ciencia de carácter marcadamente multidisciplinar-, es el único que garantiza un abordaje integral de estos pacientes en sus vertientes biológica y, de forma inseparable, como en cualquier otro trastorno mental, psicológica y social.
La Asociación Mundial de Patología Dual (WADD, por sus siglas en inglés), guiada por su presidente, el profesor Pedro Ruiz, comienza a liderar y coordinar iniciativas para integrar la salud mental y mejorar la formación, la educación y la asesoría de las administraciones alrededor del mundo. México, a través de la red asistencial Centros de Integración Juvenil, ha asumido el liderazgo en Latinoamérica.

\section{REFERENCIAS}

Arias, F., Szerman, N., Vega, P., Mesias, B., Basurte, I., Morant, C., ..., Babín, F. (2013). Estudio Madrid sobre prevalencia y características de los pacientes con patología dual en tratamiento en las redes de salud mental y de atención al drogodependiente. Adicciones, 25(2), 118-127. doi: 10.20882/adicciones.59

Belin, D., Belin-Rauscent, A., Everitt, B. J., \& Dalley, J. W. (2016). In search of predictive endophenotypes in addiction: insights from preclinical research. Genes, Brain and Behavior, 15(1), 74-88. doi: $10.1111 / \mathrm{gbb} .12265$

Chamberlain, S. R., Stochl, J., Redden, S. A., Odlaug, B. L., \& Grant, J. E. (2017). Latent class analysis of gambling subtypes and impulsive/compulsive associations: time to rethink diagnostic boundaries for gambling disorder? Addictive Behaviors, 72, 7985. doi: 10.1016/j.addbeh.2017.03.020

Lev-Ran, S., Imtiaz, S., Rehm, J., \& Le Foll, B. (2013). Exploring the association between lifetime prevalence of mental illness and transition from substance use to substance use disorders: results from the National Epidemiologic Survey of Alcohol and Related Conditions (NESARC). The American Journal on Addictions, 22(2), 93-98. doi: 10.1111/j.1521-0391.2013.00304.x

National Institute on Drug Abuse. (2007, 1 de febrero). Addiction and co-occurring mental disorders. Recuperado de https:// www.drugabuse.gov/news-events/nida-notes/2007/02/addiction -co-occurring-mental-disorders

Perna, G., \& Nemeroff, C. B. (2017). Personalized medicine in psychiatry: back to the future. Personalized Medicine in Psychiatry, 1-2, 1. doi: 110.1016/j.pmip.2017.01.001

Sociedad Española de Patología Dual. (2017). Decálogo de Patología Dual. Recuperado de http://www.patologiadual.es/docs/ Decalogo_Patologia_Dual_SEPDv2.pdf

Szerman N., \& Martinez-Raga, J. (2015) Dual disorders: two different mental disorders? Advances in Dual Diagnosis, 8(2),1-4. doi: 10.1108/ADD-03-2015-0004

Szerman, N., Martinez-Raga, J., Peris, L., Roncero, C., Basurte, I., Vega, P., ... Casas, M. (2013). Rethinking dual disorders/pathology. Addictive Disorders \& Their Treatment, 12(1), 1-10. doi: 10.1097/adt.0b013e31826e7b6a

Volkow, N. D., Koob, G. F., \& McLellan, A. T. (2016). Neurobiologic advances from the brain disease model of addiction. New England Journal of Medicine, 374(4), 363-371. doi: 10.1056/ nejmra1511480 